\title{
Hamstring Architectural and Functional Adaptations Following Long vs. Short Muscle Length Eccentric Training
}

\author{
Kenny Guex ${ }^{1}$, Francis Degache ${ }^{1 *}$, Cynthia Morisod ${ }^{2}$, Matthieu Sailly ${ }^{3}$ and \\ Gregoire P. Millet ${ }^{2}$
}

${ }^{1}$ Department of Physiotherapy, University of Health Sciences (Haute Ecole de Santé Vaud), University of Applied Sciences and Arts Western Switzerland (Haute École Spécialisée de Suisse Occidentale), Lausanne, Switzerland, ${ }^{2}$ Department of Physiology, Faculty of Biology and Medicine, Institute of Sport Sciences, University of Lausanne, Lausanne, Switzerland,

${ }^{3}$ Centre Medical Synergie, Lausanne, Switzerland

\section{OPEN ACCESS}

Edited by:

Francois Hug

University of Nantes, France

Reviewed by:

Gaël Guilhem,

Institut National du Sport et de L'Éducation Physique, France

Antoine Nordez,

University of Nantes, France

*Correspondence:

Francis Degache

francis.degache@hesav.ch

Specialty section: This article was submitted to

Exercise Physiology, a section of the journal

Frontiers in Physiology

Received: 03 February 2016

Accepted: 21 July 2016

Published: 03 August 2016

Citation:

Guex K, Degache F, Morisod C, Sailly M and Millet GP (2016)

Hamstring Architectural and

Functional Adaptations Following Long vs. Short Muscle Length

Eccentric Training.

Front. Physiol. 7:340.

doi: 10.3389/fphys.2016.00340
Most common preventive eccentric-based exercises, such as Nordic hamstring do not include any hip flexion. So, the elongation stress reached is lower than during the late swing phase of sprinting. The aim of this study was to assess the evolution of hamstring architectural (fascicle length and pennation angle) and functional (concentric and eccentric optimum angles and concentric and eccentric peak torques) parameters following a 3-week eccentric resistance program performed at long (LML) vs. short muscle length (SML). Both groups performed eight sessions of 3-5 58 slow maximal eccentric knee extensions on an isokinetic dynamometer: the SML group at $0^{\circ}$ and the $L M L$ group at $80^{\circ}$ of hip flexion. Architectural parameters were measured using ultrasound imaging and functional parameters using the isokinetic dynamometer. The fascicle length increased by $4.9 \%(p<0.01$, medium effect size) in the SML and by $9.3 \%(p<0.001$, large effect size) in the LML group. The pennation angle did not change $(p=0.83)$ in the SML and tended to decrease by $0.7^{\circ}(p=0.09$, small effect size) in the LML group. The concentric optimum angle tended to decrease by $8.8^{\circ}(p=0.09$, medium effect size) in the SML and by $17.3^{\circ}(p<0.01$, large effect size) in the LML group. The eccentric optimum angle did not change $(p=0.19$, small effect size) in the SML and tended to decrease by $10.7^{\circ}(\rho=0.06$, medium effect size) in the $L M L$ group. The concentric peak torque did not change in the SML $(p=0.37)$ and the $L M L$ $(p=0.23)$ groups, whereas eccentric peak torque increased by $12.9 \%(p<0.01$, small effect size) and $17.9 \%$ ( $p<0.001$, small effect size) in the SML and the LML group, respectively. No group-by-time interaction was found for any parameters. A correlation was found between the training-induced change in fascicle length and the change in concentric optimum angle $(r=-0.57, p<0.01)$. These results suggest that performing eccentric exercises lead to several architectural and functional adaptations. However, further investigations are required to confirm the hypothesis that performing eccentric exercises at LML may lead to greater adaptations than a similar training performed at SML.

Keywords: hamstring, eccentric, muscle length, fascicle length, pennation angle, optimum angle, strength 


\section{INTRODUCTION}

Hamstring strain injuries, and especially biceps femoris long head strain injuries, are among the most frequent injuries in sports requiring high-speed running, such as football, rugby, Australian football, Gaelic football, American football, or track and field (Brooks et al., 2005; Ekstrand et al., 2011; Elliott et al., 2011; Alonso et al., 2012; Murphy et al., 2012; Orchard et al., 2013). The long head of the biceps femoris has higher injury susceptibility at faster sprinting speed, since peak local fiber strain, fiber strain non-uniformity, and the amount of muscle undergoing larger strains are increased at faster speeds (Fiorentino et al., 2014). The late swing phase of sprinting is believed to be the main period of susceptibility to hamstring strain injuries (Chumanov et al., 2012). During this phase, the hamstring are undergoing an eccentric contraction to decelerate the knee extension, while the hip is flexed. Combined with the knee extension movement, this hip flexion position induces a substantial elongation stress on the muscle-tendon unit of the bi-articular hamstrings (elongation stress = hip flexion angle-knee flexion angle; Schache et al., 2012; Guex and Millet, 2013).

The evidence from randomized controlled trials are inconclusive to draw conclusions on the effectiveness of interventions used to prevent hamstring injuries (Goldman and Jones, 2010). The complex nature of hamstring strain injuries made that no one-single approach can be considered the gold standard for prevention (Opar et al., 2012). Nonetheless, eccentric-based intervention has been shown to be a promising method to reduce the risk of hamstring strain injuries (Askling et al., 2003; Brooks et al., 2006; Gabbe et al., 2006; Arnason et al., 2008; Petersen et al., 2011; Nichols, 2013). This may be explained by the increase in eccentric strength and by the observed shift of the optimum angle (i.e., the angle at which peak torque occurs) in the direction of longer muscle length following an eccentric strength program (Brockett et al., 2001; Askling et al., 2003; Mjolsnes et al., 2004; Clark et al., 2005; Kilgallon et al., 2007; Potier et al., 2009; Reeves et al., 2009; Brughelli et al., 2010; Martínez-Ruiz et al., 2014; Guex et al., 2016; Timmins et al., 2016a). Along with the increase in strength, eccentric-based interventions induce neuro-muscular adaptations, including improvement in the neural factors, muscle hypertrophy and an increase in fascicle pennation angle, suggesting an addition of sarcomeres in parallel (Moritani and deVries, 1979; Kawakami et al., 1993; Aagaard et al., 2001; Reeves et al., 2004; Blazevich et al., 2007; Seynnes et al., 2007). Whilst there is a general consensus that increases in pennation are driven by hypertrophy, a lack of relationship between changes in muscle size and changes in pennation can be observed. In line, with this unclear relationship, two studies have shown no modification or a slight decrease in pennation angle of the biceps femoris following eccentric training (Potier et al., 2009; Timmins et al., 2016a). The shift of the optimum angle, is often attributed to an increase in fascicle length, suggesting an addition of sarcomeres in series within the muscle, which enables operating over a greater range of motion without overstretch (Blazevich et al., 2007; Seynnes et al., 2007; Potier et al., 2009; Reeves et al., 2009). But, to date no research has examined the direct effect of a change in fascicle lengths on the risk of injury (Timmins et al., 2016b). Other contraction modes, such as the isometric, can also cause angle-specific adaptations and shift in optimum angle (Kitai and Sale, 1989). This may be explained by the fact that other mechanisms, such as neural factors may also influence the force-length relationship. Indeed, an improvement in rate of force development would also contribute to a shift in $\mathrm{OA}$ in the direction of longer muscle length (Aagaard et al., 2002). Moreover, recent detailed evidence suggest a possible role of region-specific muscle hypertrophy in addition to a neural mechanism (Noorkoiv et al., 2014).

In order to optimize the hamstring strength exercises in a perspective of injuries prevention, a conceptual framework based on the biomechanical parameters of sprinting has been recently proposed (Guex and Millet, 2013). It is suggested to use eccentric contractions performed at a slow to moderate angular velocity with a movement focused at the knee joint, while the hip is kept in a large flexion position in order to reach an elongation stress of the hamstrings greater than in the late swing phase (Guex and Millet, 2013). The most common eccentricbased interventions, which were shown to be efficient to reduce hamstring strain injuries used either the Nordic hamstring exercise (Brooks et al., 2006; Gabbe et al., 2006; Arnason et al., 2008; Petersen et al., 2011; Nichols, 2013), or the yo-yo hamstring curl exercise (Askling et al., 2003). These two exercises involve eccentric contractions performed at a slow to moderate angular velocity with a movement focused at the knee joint. However, they include only minimal hip flexion. Then, the elongation stress reached at the end of these two movements is close to $0\left(<30^{\circ}\right.$ of hip flexion $-0^{\circ}$ of knee flexion $)$, while it is widely positive during the late swing phase $\left(>70^{\circ}\right.$ of hip flexion$<30^{\circ}$ of knee flexion; Novacheck, 1998; Thelen et al., 2005). The elongation stress seems a relevant injury risk factor: the magnitude of musculotendinous lengthening occurring during repeated eccentric contractions was related to the severity of the subsequent muscle damage (Lieber and Friden, 1993). Moreover, some evidence have reported that the training range of motion (i.e., muscle excursion range during loading) could be the dominant stimulus for adaptations in fascicle length (Blazevich et al., 2007).

To our knowledge, it is unknown how the hamstring would adapt following an eccentric resistance training performed at long (i.e., positive elongation stress) vs. short (i.e., negative elongation stress) muscle length. Then, the aim of this study was to assess the change in: (1) hamstring architectural parameters (fascicle length and pennation angle of the long head of the biceps femoris); (2) hamstring functional parameters (concentric and eccentric optimum angles and concentric and eccentric peak torques) following a 3-week eccentric resistance program performed at long (LML) vs. short muscle length (SML). Since muscle architectural adaptations have been shown to rapidly occur (Blazevich et al., 2007; Seynnes et al., 2007; Timmins et al., 2016a), it was hypothesized that the proposed eccentric training would increase the fascicle length, the pennation angle and the strength, and would decrease the optimum angle in both groups. Furthermore, it was hypothesized that architectural 
and functional adaptations would be greater following eccentric training performed at LML vs. SML.

\section{MATERIALS AND METHODS}

\section{Subjects}

Twenty-two subjects were recruited voluntarily to the study and randomly allocated into two equal sized groups: the short (SML) and long (LML) muscle length groups. Table 1 summarizes the characteristics of the two groups, which were similar in age $(p=0.55)$, height $(p=0.47)$, and body mass $(p=0.72)$. The subjects performed only recreational physical activity. They were excluded if they reported traumatological disorders, history of hip or knee pathology or dysfunction. Prior to the beginning of the study, the subjects signed an informed consent after explanation of the study protocol, data collection procedures, significance of the study objectives, benefits, and risks of the investigation. Ethical approval for the project was obtained from the local committee on human research (Commission cantonale d'éthique de la recherche sur l'être humain, CCER-VD, Agreement 181/15, Lausanne, Switzerland).

\section{Experimental Design}

For 3-weeks, the subjects completed eight hamstring eccentric sessions on an isokinetic dynamometer (Biodex System 2, Biodex Medical Systems, Shirley, New York, USA). In order to modify the hamstring muscle length, the SML group performed all training sessions in a supine position and the LML in a seated position. Ultrasound and isokinetic measurements were performed before the first training session and 4 days after the last training session in order to assess the evolution of: (1) hamstring architectural parameters: fascicle length (FL) and pennation angle (PA) of the long head of the biceps femoris; (2) hamstring functional parameters: optimum angle in concentric at $60^{\circ} / \mathrm{s}(\mathrm{ConOA})$, optimum angle in eccentric at $30^{\circ} / \mathrm{s}(\mathrm{EccOA})$, concentric peak torque at $60 \%$ (ConPT), and eccentric peak torque at $30^{\circ} / \mathrm{s}(\mathrm{EccPT})$.

\section{Eccentric Resistance Training}

Prior to each session, the subjects performed a 10-min warmup on a cycling ergometer $(60 \mathrm{rpm}, 80$ watts). They were then placed on the isokinetic dynamometer. Stabilization straps were positioned across their chest, pelvis, and thigh. The lever arm shin-pad was positioned just proximal to the lateral malleolus. For practical reasons, the subjects were all trained and tested on their right limb.

TABLE 1 | Subject's characteristics (mean $\pm S D$ ).

\begin{tabular}{lcc}
\hline & SML group $(\boldsymbol{n}=\mathbf{1 1})$ & LML group $(\boldsymbol{n}=\mathbf{1 1})$ \\
\hline Female/male ratio & $6 / 5$ & $6 / 5$ \\
Age, years & $27.3 \pm 3.9$ & $28.4 \pm 4.5$ \\
Height, cm & $173.5 \pm 10.8$ & $170.7 \pm 5.9$ \\
Weight, kg & $66.0 \pm 13.6$ & $64.0 \pm 12.7$ \\
\hline
\end{tabular}

SML, short muscle length; LML, long muscle length.
The eccentric resistance training consisted in eight sessions performed during 3-weeks: two sessions per week the 1st week and three sessions in second and 3rd weeks. Each session consisted of three (weeks 1), four (week 2), or five (week 3) sets of eight maximal eccentric knee extensions performed at $30 \%$ on the isokinetic dynamometer. A 3-min rest period was allocated between each set. For each repetition, the knee range of motion was fixed at $110^{\circ}$ (between 110 and $0^{\circ}$ of knee flexion, $0^{\circ}$ corresponding to full extension).

Both SML and LML groups followed the same training program. However, in order to modify the hamstring muscle length, the SML group performed the resistance training at $0^{\circ}$ of hip flexion (i.e., supine position; Figure 1A) and the LML group at $80^{\circ}$ of hip flexion (i.e., seated position; Figure 1B). Thus, during each repetition, the elongation stress moved from -110 to 0 in the SML group, while it moved from -30 to 80 in the LML group (Guex and Millet, 2013).

\section{Architectural Adaptations Assessment}

Architectural parameters were assessed using ultrasound imaging, which was shown to be a reliable method for measuring FL and PA of the long head of the biceps femoris (Chleboun et al., 2001). Subjects were positioned in a prone position with their knee fully extended and their muscles relaxed. The probe (42 $\mathrm{mm}$ linear array transducer, $10 \mathrm{MHz}$ wave frequency) of the ultrasonic instrument (SSD-2000, ALOKA, Tokyo, Japan) was placed directly on the skin above the middle-belly of the long head of the biceps femoris with transmission gel to obtain acoustic coupling. The probe was oriented parallel to the muscle fascicles and perpendicular to the skin. Once the probe was appropriately placed, its position was marked on the skin in order to replace it in the same position after the 3-weeks training program. After each training session, the mark was controlled and redrawn if necessary.

FL was measured by manually outlining visible parts of the muscle fascicle, which crossed the midpoint between the superficial and deep aponeurosis in the center of the ultrasound image. The length of the missing portions was estimated by measuring the linear distances from the identifiable ends of a fascicle to the intersection between the line drawn from the fascicle and the line drawn from the deep to the superficial aponeurosis (Blazevich et al., 2007). The angle between the line marking the deep aponeurosis and the outlined fascicle corresponded to the PA (Figure 2). Test-retest of architectural parameters assessment on three consecutive days in 10 control subjects indicated FL and PA measurements had a coefficient of variation $(\mathrm{CV})$ of $2.1 \%(\sim 1.8 \mathrm{~mm})$ and $2.3 \%\left(\sim 0.32^{\circ}\right)$, respectively.

\section{Functional Adaptations Assessment}

To assess the functional parameters, maximal concentric and eccentric strength of the right hamstring was measured using the isokinetic dynamometer. Pre- and post-training assessments took place at the same time of the day. The dynamometer was calibrated according to the manufacturer's recommendations and following the instructions for optimal reproducibility. Prior to the testing procedures, the subjects performed a similar warm-up 

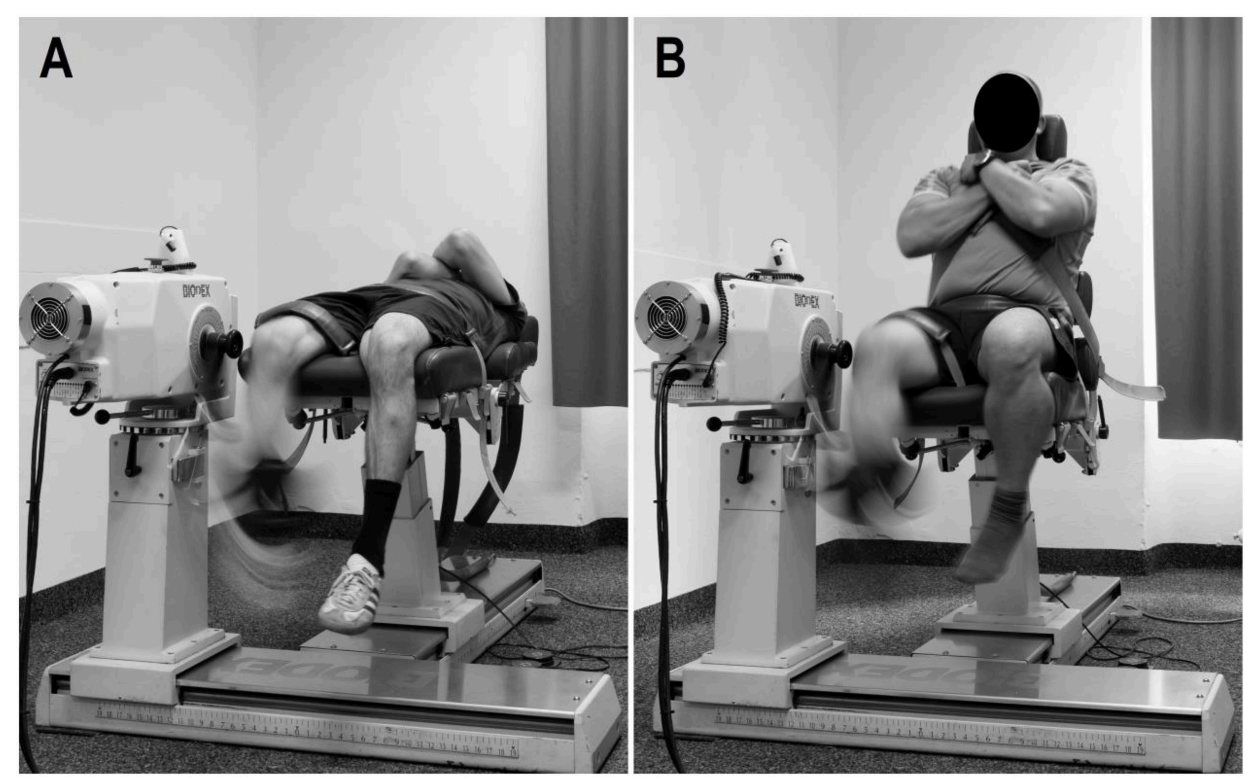

FIGURE 1 | Hamstring eccentric resistance training at (A) short and (B) long muscle length.

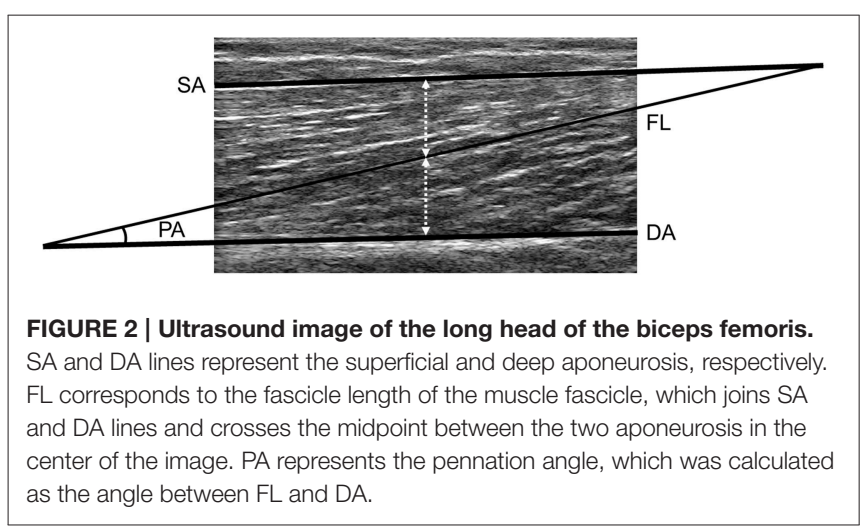

as described in the eccentric resistance training part. They were then correctly placed on the dynamometer. The hip flexion angle was held at $80^{\circ}$ (i.e., seated position) and the knee range of motion was similar as described in the eccentric resistance training part. The $80^{\circ}$ hip flexion position was chosen in order to test the hamstring of each subject at a sprint specific angle (Guex et al., 2012; Guex and Millet, 2013). Before testing, gravity correction was obtained by measuring the torque exerted on the lever arm shin-pad with the knee in extension in a relaxed state. Prior to the pre-training assessment, familiarization sets were performed, consisting in 10 progressive concentric knee flexions at $60^{\circ} / \mathrm{s}$ followed by 10 progressive eccentric knee extensions at $30 \%$ with a 1-min rest period between both sets.

The isokinetic test consisted of a set of six maximal concentric knee flexions at $60^{\circ} / \mathrm{s}$ followed by six maximal eccentric knee extensions at $30 \%$ with a 3 -min rest period between both sets. $\mathrm{OA}$ and PT were determined by fitting a 4th order polynomial curve to the raw data of the best repetition. OA corresponded to the angle at which the PT was reached. In addition, to complete the analysis on the torque-angle relationship, mean concentric and eccentric torques between $10^{\circ}-20^{\circ}, 20^{\circ}-30^{\circ}, 30^{\circ}$ $40^{\circ}, 40^{\circ}-50^{\circ}, 50^{\circ}-60^{\circ}, 60^{\circ}-70^{\circ}, 70^{\circ}-80^{\circ}, 80^{\circ}-90^{\circ}$, and $90^{\circ}-$ $100^{\circ}$ of knee flexion were recorded at pre- and post-training assessments.

\section{Statistical Analysis}

Data are expressed as mean \pm standard deviation $(S D)$. They were screened for a normal distribution using Shapiro-Wilk normality tests. To assess assumptions of variance, Mauchly's test of sphericity was performed. In order to observe the evolution of architectural and functional parameters following the eccentric resistance training, two-way (group $\times$ time) analysis of variance (ANOVA) with repeated measures were used. Tukey post-hoc tests were used to localize the differences between means. The importance of the differences found between pre- and posttraining assessment were assessed through the effect size and Cohen's $d$ coefficient (Cohen, 1988), interpreted as follows: small difference: $0.15 \leq d<0.40$, medium difference: $0.40 \leq d<0.75$, large difference: $0.75 \leq d<1.10$, and very large difference: $d \geq$ 1.10. Finally, the relationship between changes in architectural and functional adaptations were examined using a Pearson correlation coefficients, interpreted as follows: weak: $0.20 \leq r<$ 0.40 , moderate: $0.40 \leq r<0.60$, good: $0.60 \leq r<0.80$ and excellent: $0.80 \leq r \leq 1.00$. For all statistical analysis, significance was set at $p<0.05$. Statistical analysis were performed with SigmaPlot 12.5 (Systat Software Inc., San Jose, CA).

\section{RESULTS}

The values of architectural and functional parameters before and after the eccentric resistance training are presented in Table 2. At 
TABLE 2 | Hamstring architectural and functional parameters (mean $\pm S D$ ) before (Pre-) and after (Post-) the eccentric resistance training performed at short vs. long muscle length.

\begin{tabular}{|c|c|c|c|c|c|c|c|c|}
\hline & \multicolumn{4}{|c|}{ SML group $(n=11)$} & \multicolumn{4}{|c|}{ LML group $(n=11)$} \\
\hline & Pre- & Post- & Net change $[95 \% \mathrm{Cl}]$ & Effect size & Pre- & Post- & Net change $[95 \% \mathrm{Cl}]$ & Effect size \\
\hline \multicolumn{9}{|c|}{ ARCHITECTURAL PARAMETERS } \\
\hline $\mathrm{FL}, \mathrm{mm}$ & $84.1 \pm 7.3$ & $88.2 \pm 7.9^{\star \star}$ & $4.1[2.5 ; 5.7]$ & 0.57 (medium) & $82.0 \pm 9.3$ & $89.4 \pm 8.13^{\star \star \star}$ & $7.4[4.5 ; 10.2]$ & 0.89 (large) \\
\hline $\mathrm{PA},{ }^{\circ}$ & $15.0 \pm 2.9$ & $14.9 \pm 2.2$ & $-0.1[0.7 ;-0.9]$ & 0.04 & $14.6 \pm 3.4$ & $13.8 \pm 3.0$ & $-0.7[0.1 ;-1.5]$ & 0.24 (small) \\
\hline \multicolumn{9}{|c|}{ FUNCTIONAL PARAMETERS } \\
\hline $\mathrm{ConOA}^{\circ}$ & $77.0 \pm 16.5$ & $68.3 \pm 18.7$ & $-8.8[-1.6 ;-15.9]$ & 0.52 (medium) & $78.8 \pm 14.8$ & $61.5 \pm 24.49^{\star \star}$ & $-17.3[-5.6 ;-29.0]$ & 0.90 (large) \\
\hline EccOA, ${ }^{\circ}$ & $40.0 \pm 21.8$ & $32.7 \pm 25.1$ & $-7.2[0.9 ;-15.4]$ & 0.32 (small) & $40.6 \pm 19.3$ & $30.0 \pm 17.2$ & $-10.7[1.8 ;-23.1]$ & 0.61 (medium) \\
\hline ConPT, Nm & $47.4 \pm 16.9$ & $49.0 \pm 16.1$ & $1.7[-1.5 ; 4.8]$ & 0.11 & $43.1 \pm 17.8$ & $45.3 \pm 19.9$ & $2.3[-1.7 ; 6.2]$ & 0.13 \\
\hline EccPT, Nm & $59.4 \pm 22.9$ & $65.5 \pm 21.0^{\star \star}$ & $6.1[3.6 ; 8.7]$ & 0.29 (small) & $52.1 \pm 23.9$ & $60.1 \pm 25.3^{\star \star}$ & $8.0[3.7 ; 12.3]$ & 0.34 (small) \\
\hline
\end{tabular}

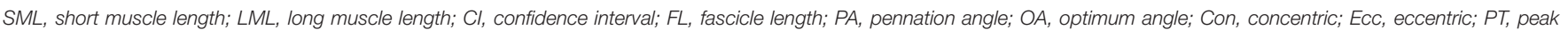
torque. ${ }^{* \star} p<0.01$ and ${ }^{* \star *} p<0.001$ for differences with pre-training.

pre-, no difference in FL $(p=0.56)$, PA $(p=0.72)$, ConOA $(p=0.83), \operatorname{EccOA}(p=0.94)$, ConPT $(p=0.58)$ and EccPT ( $p=0.47$ ) was observed between the SML and the LML groups.

\section{Architectural Adaptations}

Following the eccentric resistance training, the FL increased by $4.9 \%(p<0.01$, medium effect size $)$ in the SML and by $9.3 \%$ $(p<0.001$, large effect size) in the LML group. The PA did not change $(p=0.83)$ in the SML and tended to decrease by $0.7^{\circ}$ ( $p=0.09$, small effect size) in the LML group. No group-by-time interaction was found for FL $(p=0.74)$ and PA $(p=0.39)$.

\section{Functional Adaptations}

The ConOA tended to decrease by $8.8^{\circ}(p=0.09$, medium effect size) in the SML and by $17.3^{\circ}(p<0.01$, large effect size) in the LML group. The EccOA did not change $(p=0.19$, small effect size) in the SML and tended to decrease by $10.7^{\circ}(p=0.06$, medium effect size) in the LML group. The ConPT did not change in the SML $(p=0.37)$ and the LML $(p=0.23)$ groups, whereas EccPT increased by $12.9 \%$ ( $p<0.01$, small effect size) and $17.9 \%$ ( $p<0.001$, small effect size) in the SML and the LML group, respectively. No group-by-time interaction was found for ConOA $(p=0.41)$, EccOA $(p=0.76)$, ConPT $(p=0.63)$, and $\operatorname{EccPT}(p=0.59)$.

Following the 3-week eccentric program, the mean concentric torque at $60^{\circ} \mathrm{s}$ significantly increased by $23.3 \%(p<0.05$, small effect size) and $42.0 \%$ ( $p<0.01$, medium effect size) between $10^{\circ}-20^{\circ}$, by $17.1 \%$ ( $p<0.05$, small effect size) and $31.4 \%$ ( $p<0.01$, medium effect size) between $20^{\circ}-30^{\circ}$, by $14.4 \%$ $(p<0.05$, small effect size) and $24.1 \%(p<0.01$, small effect size) between $30^{\circ}-40^{\circ}$, by $12.5 \%(p<0.05$, small effect size) and $16.9 \%$ ( $p<0.01$, small effect size) between $40^{\circ}-50^{\circ}$ and by $10.3 \%$ ( $p<0.05$, small effect size) and $11.1 \%(p<0.05$, small effect size) between $50^{\circ}-60^{\circ}$ in the SML and the LML group, respectively (Figure $\mathbf{3 A}$ ). The mean eccentric torque at $30 \%$ significantly increased by $10.4 \%(p<0.05$, small effect size) and $22.6 \%\left(p<0.001\right.$, medium effect size) between $10^{\circ}$ $20^{\circ}$, by $8.8 \%(p<0.05$, small effect size $)$ and $19.1 \%(p<0.001$, medium effect size) between $20^{\circ}-30^{\circ}$ in the SML and the LML group, respectively. Moreover, it only significantly increased in LML group by $15.4 \%$ ( $p<0.01$, small effect size) and $11.7 \%$ $\left(p<0.05\right.$, small effect size) between $30^{\circ}-40^{\circ}$ and $40^{\circ}-50^{\circ}$, respectively (Figure 3B). At each knee angle, no group-by-time interaction was found for both concentric and eccentric mean torques.

\section{Relationship between Architectural and Functional Adaptations}

A moderate negative correlation was found between the traininginduced change in FL and the change in ConOA $(r=-0.57$, $p<0.01)$.

\section{DISCUSSION}

The aim of this study was to assess the changes in hamstring architectural and functional parameters following a 3-week eccentric resistance training performed at LML vs. SML. In line with our hypothesis, the FL and the EccPT significantly increased in both groups, the ConOA significantly decreased in the LML group and tended to decrease in the SML group, while the EccOA tended to decreased in the LML group. However, two parameters did not significantly change from pre- to posttraining assessment: the PA and the ConPT. Furthermore, it was hypothesized that architectural and functional adaptations would be greater in the LML group than in the SML group. It is interesting to observe that for the changes in FL, ConOA, EccOA, mean concentric torque between $10^{\circ}$ and $20^{\circ}$ and between $20^{\circ}$ and $30^{\circ}$ and mean eccentric torque between $10^{\circ}$ and $20^{\circ}$ and between $20^{\circ}$ and $30^{\circ}$, the effect sizes were greater in the LML than in the SML group. However, no group-by-time interaction was found. Further investigations are then required to confirm the hypothesis that performing eccentric exercises at a long muscle length could lead to greater adaptations than exercises performed with lesser hamstring elongation stress.

The mean FL- and PA-values of the long head of the biceps femoris observed at pre-training assessment $\left(\sim 83 \mathrm{~mm}\right.$ and $\sim 15^{\circ}$, respectively) are in line with previous studies $(\sim 58-117 \mathrm{~mm}$ 
and $\sim 13^{\circ}-19^{\circ}$, respectively; Chleboun et al., 2001; Woodley and Mercer, 2005; Potier et al., 2009; Kellis et al., 2010; Timmins et al., 2016a), showing the robustness of the present measurements. Following the 3-week eccentric program performed at short and long muscle length, the FL significantly increased by $\sim 5$ and $9 \%$, respectively. These modifications are considerably lower than the $\sim 34 \%$ increase observed by Potier et al. (2009) following an 8 -week eccentric program performed in a prone position on a hamstring leg curl machine (i.e., at SML). They are also lower than the $15 \%$ increase obtained by Timmins et al., after 14 and 21 days of eccentric training on an isokinetic dynamometer with a protocol comparable to our intervention at long muscle length (Timmins et al., 2016a). Our results are consistent with those of Seynnes et al., who have reported $\sim 2$, 6 , and $10 \%$ increases in vastus lateralis FL after 10, 20, and 35 days of eccentric training at long muscle length, respectively, or with Blazevich et al., who have found a $\sim 3 \%$ increase in vastus lateralis FL following a 10 -week eccentric program performed at long muscle length (Blazevich et al., 2007; Seynnes et al., 2007). In this later study, the authors have found a $\sim 6 \%$ increase in vastus lateralis FL following a similar 10week program performed in concentric, suggesting that, beyond the contraction mode, it is the training range of motion (or muscle excursion range) that is paramount for fascicle length adaptation. To our knowledge, the present study is the first one attempting to test this hypothesis by comparing two similar training interventions performed at different muscle lengths. Although the effect size was greater following the intervention performed at long muscle length, there was no group-by-time interaction. To show a significant difference $(p<0.05)$ between the increase in FL obtained in the LML $(7.4 \mathrm{~mm})$ and SML $(4.1 \mathrm{~mm})$ groups with $80 \%$ power, the sample size should be of 28 subjects in each group. This is an important limitation of the present study.

Following the 3-week eccentric program, the PA did not change in the SML group and tended to slightly decrease in the LML group. One may assume that this later results are the consequence of the lack of hypertrophy due to the short intervention, since eccentric training has classically been associated to an increase in PA, suggesting an addition of sarcomeres in parallel (Kawakami et al., 1993; Aagaard et al., 2001; Blazevich et al., 2007; Seynnes et al., 2007). However, Timmins et al. found a significant slight decrease in PA after only 14 days of hamstring eccentric training (Timmins et al., 2016a), and Potier et al., as in the present the study, reported a slight but not significant decrease in PA after their eccentric intervention on the hamstring (Potier et al., 2009). Potier et al. stated that any change in PA may be muscle specific, and that significant changes may have been observed in other knee flexor muscles, such as the semitendinosus.

After the intervention performed at long muscle length, the ConOA shifted by $17^{\circ}$ in the direction of longer muscle length, while the EccOA tended to shift by $11^{\circ}$. At SML, only the ConOA tended to shift by $9^{\circ}$. These shifts are in line with previous studies on the hamstring $\left(4^{\circ}-21^{\circ}\right.$; Clark et al., 2005; Kilgallon et al., 2007; Brughelli et al., 2010; Martínez-Ruiz et al., 2014). A decrease in $\mathrm{OA}$ is classically attributed to an increase in fascicle length,

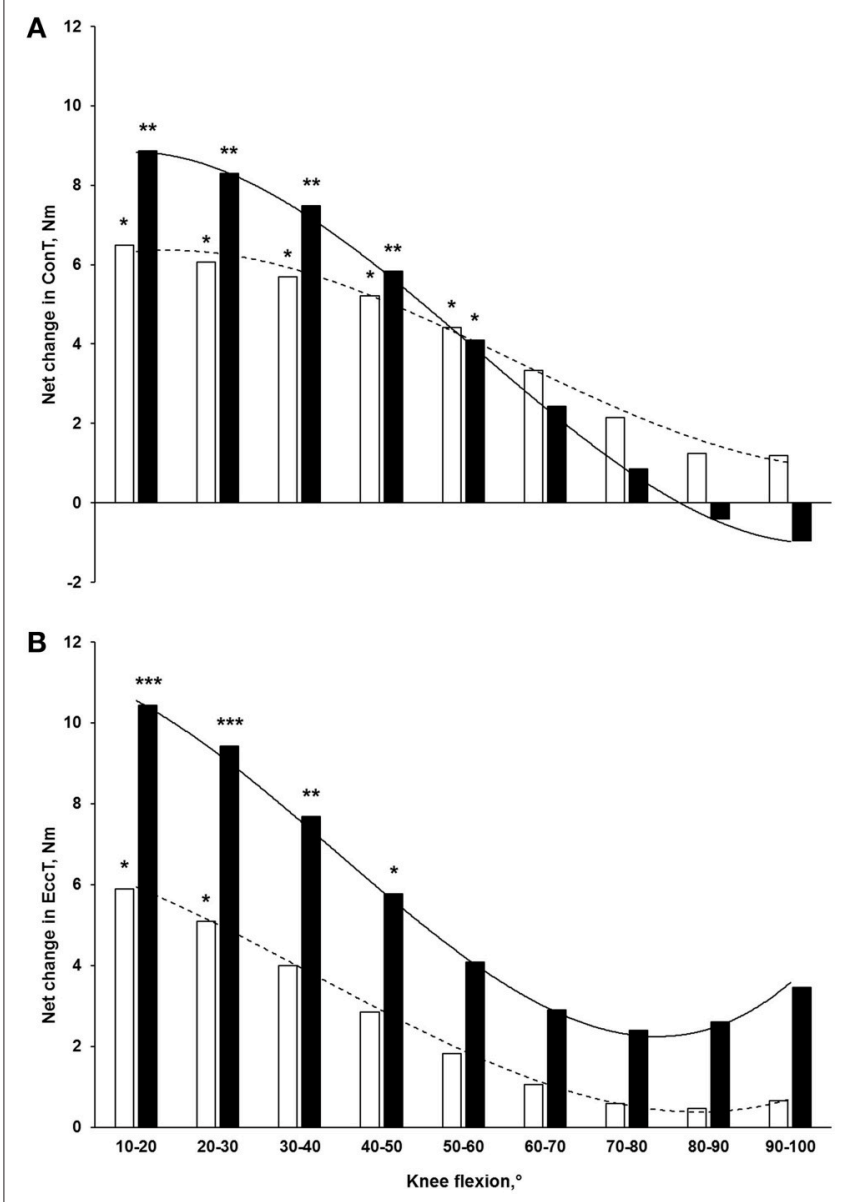

FIGURE 3 | Net changes in mean torques at different knee flexions in (A) concentric at $60 \%$ s and (B) eccentric at $30 \%$ s following a 3-week eccentric program. Light and solid blocks represent the SML and LML groups, respectively. ${ }^{*} p<0.05,{ }^{* *} p<0.01$, and ${ }^{* *} p<0.001$ for differences with pre-training.

suggesting an addition of sarcomeres in series within the muscle (Blazevich et al., 2007; Seynnes et al., 2007; Potier et al., 2009; Reeves et al., 2009). Interestingly, this hypothesis is supported by the correlation found in the present study between the increase in FL and the shift in ConOA in the direction of longer muscle length, suggesting that the increase in fascicle length allows the muscle to operate effectively over a greater range of motion. However, one must be cautious when interpreting the relation between architectural and functional parameters, since no correlation was found between the increase in FL and the shift in EccOA ( $r=-0.17, p=0.46)$. This may be explained by the fact that other factors, such as neural mechanism and/or region-specific muscle hypertrophy may also have influenced the force-length relationship. It is likely that the shift in OA is a multifactorial event. Nonetheless, the data presented in Figure 3 highlights the effect of the eccentric training on hamstring function: both concentric and eccentric torques preferentially increased at long muscle length (i.e., when the knee is in a low flexion position) with a greater training-induced adaptation in 
the LML group. This may be particularly relevant for injuries prevention in sprinters, since the late swing phase of sprinting has been shown to be the main period of susceptibility to hamstring strain injuries: during this phase, the hamstring are stretched by $\sim 10 \%$ beyond their upright length (Chumanov et al., 2012; Schache et al., 2012). Thus, an eccentric intervention would help the hamstring to work more efficiently without overstretch during the whole sprinting cycle.

In both groups, the EccPT was significantly increased by more than $10 \%$ following the intervention, while the ConPT did not change. This large increase in eccentric strength demonstrates the efficiency of the present protocol. As discussed above, the PA did not increase in both groups, which could suggests a lack of hypertrophy. Then, the present results suggest that the increase in eccentric strength could be attributed to an improvement in the neural factors or in excitation-contraction coupling (Moritani and deVries, 1979; Warren et al., 2001; Reeves et al., 2004). However, an assessment of the electromyographic activity of the biceps femoris long head would have been necessary to confirm this hypothesis. The lack of improvement in ConPT after the isokinetic eccentric program is in line with previous studies and confirms the concept of mode specificity in isokinetic training (Tomberlin et al., 1991; Seger et al., 1998). Finally, one cannot rule out that the seated position allowed slightly greater torques in training (Guex et al., 2012). Then the small, non-significant additional changes seen in LML group might be associated with the greater training load, rather than the muscle length used in training.

The present study has some limitations. First, the proposed intervention consisted in only eight sessions over 3-weeks. Further, studies are required to investigate if larger differences between the LML and SML groups would occur with longer training period. While PA would probably have increased due to an increase in the amount of contractile tissue, one may though that FL would not have increased more, since it was shown that FL increase occurs mainly within the 1st weeks of training (Blazevich et al., 2007; Timmins et al., 2016a). Second, the LML group performed the resistance training at $80^{\circ}$ of hip flexion. Thus, during each repetition, the elongation stress moved from -30 to 80 , which is greater than the SML group $(-110$ to 0$)$. However, one may think that the elongation stress reached in the LML group could not be enough to generate an hamstring overstretch in all subjects, especially in the more flexible ones. In future investigations, it could be relevant to individualize the range of motion of the LML group in

\section{REFERENCES}

Aagaard, P., Andersen, J. L., Dyhre-Poulsen, P., Leffers, A. M., Wagner, A., Magnusson, S. P., et al. (2001). A mechanism for increased contractile strength of human pennate muscle in response to strength training: changes in muscle architecture. J. Physiol. 534(Pt 2), 613-623. doi: 10.1111/j.1469-7793.2001.t011-00613.x

Aagaard, P., Simonsen, E. B., Andersen, J. L., Magnusson, P., and Dyhre-Poulsen, P. (2002). Increased rate of force development and neural drive of human skeletal muscle following resistance training. J. Appl. Physiol. 93, 1318-1326. doi: 10.1152/japplphysiol.00283.2002 regards to the flexibility of each subject to ensure a sufficient musculotendinous elongation stress. Another limitation of the present investigation concerns the assessment of the FL. Indeed, the length of the missing portions was estimated, which implies an important extrapolation. However, the reliability testing indicated FL measurements had a CV of $2.1 \%(\sim 1.8 \mathrm{~mm})$, which is $\sim 2$ and 4 times lower than the observed FL increase in SML and LML groups, respectively. Moreover, as previously mentioned, the FL-values of the present investigation are in line with those reported in previous studies (Chleboun et al., 2001; Woodley and Mercer, 2005; Potier et al., 2009; Kellis et al., 2010; Timmins et al., 2016a). Another potential limitation, is that no control group was included in the present study design. Therefore, one may not exclude that part of the observed adaptations could be due to non-controlled factors, even if the protocol (e.g., training loads, testing procedure, ...) was perfectly controlled for each subject. Future controlled studies, are then required to reinforce the present findings. Finally, only the long head of the biceps femoris was analyzed. It is possible that the other knee flexor muscles would have responded differently to the present training protocol.

In conclusion, this study, which was the first one to investigate the influence of muscle length during eccentric training on hamstring architectural and functional parameters, reported eccentric strength, fascicle length, concentric, and eccentric optimum angles increased following eccentric intervention in both positions with no group-by-time interaction. However, fascicle length, concentric, and eccentric optimum angles increased with larger effect size following eccentric training at long than at SML. Further investigations, such as randomized controlled trials with larger sample size are required to confirm the hypothesis that performing eccentric exercises with a large elongation stress may lead to greater architectural and functional adaptations than a similar training performed at SML.

\section{AUTHOR CONTRIBUTIONS}

Conceived and designed the experiments: KG, FD, MS, and GM. Performed experiments: KG, FD, and CM. Analyzed data: KG and CM. Interpreted results of research: KG, FD, MS, and GM. Drafted manuscript and prepared tables/figures: KG, FD, and CM. Edited, critically revised paper and approved final version of manuscript: KG, FD, CM, MS, and GM. All authors have agreed to be accountable for all aspects of the work related to its accuracy and integrity. 
eccentric overload. Scand. J. Med. Sci. Sports 13, 244-250. doi: 10.1034/j.16000838.2003.00312.x

Blazevich, A. J., Cannavan, D., Coleman, D. R., and Horne, S. (2007). Influence of concentric and eccentric resistance training on architectural adaptation in human quadriceps muscles. J. Appl. Physiol. 103, 1565-1575. doi: 10.1152/japp lphysiol.00578.2007

Brockett, C. L., Morgan, D. L., and Proske, U. (2001). Human hamstring muscles adapt to eccentric exercise by changing optimum length. Med.Sci. Sports Exerc. 33, 783-790. doi: 10.1097/00005768-200105000-00017

Brooks, J. H., Fuller, C. W., Kemp, S. P., and Reddin, D. B. (2005). Epidemiology of injuries in English professional rugby union: part 1 match injuries. Br. J. Sports Med. 39, 757-766. doi: 10.1136/bjsm.2005.018135

Brooks, J. H., Fuller, C. W., Kemp, S. P., and Reddin, D. B. (2006). Incidence, risk, and prevention of hamstring muscle injuries in professional rugby union. Am. J. Sports Med. 34, 1297-1306. doi: 10.1177/03635465052 86022

Brughelli, M., Mendiguchia, J., Nosaka, K., Idoate, F., Arcos, A. L., and Cronin, J. (2010). Effects of eccentric exercise on optimum length of the knee flexors and extensors during the preseason in professional soccer players. Phys. Ther. Sport 11, 50-55. doi: 10.1016/j.ptsp.2009.12.002

Chleboun, G. S., France, A. R., Crill, M. T., Braddock, H. K., and Howell, J. N. (2001). In vivo measurement of fascicle length and pennation angle of the human biceps femoris muscle. Cells Tissues Organs 169, 401-409. doi: 10.1159/0 00047908

Chumanov, E. S., Schache, A. G., Heiderscheit, B. C., and Thelen, D. G. (2012). Hamstrings are most susceptible to injury during the late swing phase of sprinting. Br. J. Sports Med. 46, 90. doi: 10.1136/bjsports-2011-0 90176

Clark, R., Bryant, A., Culgan, J. P., and Hartley, B. (2005). The effects of eccentric hamstring strength training on dynamic jumping performance and isokinetic strength parameters: a pilot study on the implications for the prevention of hamstring injuries. Phys. Ther. Sport 6, 67-73. doi: 10.1016/j.ptsp.2005.02.003

Cohen, J. (1988). Statistical Power Analysis for the Behavioral Sciences, 2nd Edn. Hillsdale, NJ: Lawrence Erlbaum Associates.

Ekstrand, J., Hagglund, M., and Walden, M. (2011). Injury incidence and injury patterns in professional football: the UEFA injury study. Br. J. Sports Med. 45, 553-558. doi: 10.1136/bjsm.2009.060582

Elliott, M. C., Zarins, B., Powell, J. W., and Kenyon, C. D. (2011). Hamstring muscle strains in professional football players: a 10-year review. Am. J. Sports Med. 39, 843-850. doi: 10.1177/0363546510394647

Fiorentino, N. M., Rehorn, M. R., Chumanov, E. S., Thelen, D. G., and Blemker, S. S. (2014). Computational models predict larger muscle tissue strains at faster sprinting speeds. Med. Sci. Sports Exerc. 46, 776-786. doi: 10.1249/MSS.0000000000000172

Gabbe, B. J., Branson, R., and Bennell, K. L. (2006). A pilot randomised controlled trial of eccentric exercise to prevent hamstring injuries in community-level Australian Football. J. Sci. Med. Sport 9, 103-109. doi: 10.1016/j.jsams.2006. 02.001

Goldman, E. F., and Jones, D. E. (2010). Interventions for preventing hamstring injuries. Cochrane Database Syst. Rev. CD006782. doi: 10.1002/14651858. CD006782.pub2

Guex, K. J., Lugrin, V., Borloz, S., and Millet, G. P. (2016). Influence on strength and flexibility of a swing phase-specific hamstring eccentric program in Sprinters' general preparation. J. Strength Cond. Res. 30, 525-532. doi: 10.1519/JSC.0000000000001103

Guex, K., and Millet, G. P. (2013). Conceptual framework for strengthening exercises to prevent hamstring strains. Sports Med. 43, 1207-1215. doi: 10.1007/s40279-013-0097-y

Guex, K., Gojanovic, B., and Millet, G. P. (2012). Influence of hip-flexion angle on hamstrings isokinetic activity in sprinters. J. Athl. Train. 47, 390-395. doi: 10.4085/1062-6050-47.4.04

Kawakami, Y., Abe, T., and Fukunaga, T. (1993). Muscle-fiber pennation angles are greater in hypertrophied than in normal muscles. J. Appl. Physiol. 74, 2740-2744.

Kellis, E., Galanis, N., Natsis, K., and Kapetanos, G. (2010). Muscle architecture variations along the human semitendinosus and biceps femoris (long head) length. J. Electromyogr. Kinesiol. 20, 1237-1243. doi: 10.1016/j.jelekin.2010.07.012
Kilgallon, M., Donnelly, A. E., and Shafat, A. (2007). Progressive resistance training temporarily alters hamstring torque-angle relationship. Scand. J. Med. Sci. Sports 17, 18-24. doi: 10.1111/j.1600-0838.2005. 00491.x

Kitai, T. A., and Sale, D. G. (1989). Specificity of joint angle in isometric training. Eur. J. Appl. Physiol. Occup. Physiol. 58, 744-748. doi: 10.1007/BF0063 7386

Lieber, R. L., and Friden, J. (1993). Muscle damage is not a function of muscle force but active muscle strain. J. Appl. Physiol. 74, 520-526.

Martínez-Ruiz, E., Mendiguchia, J., Rubio-Arias, J. A., Alcaraz, P. E., and EsparzaRos, F. (2014). "Effect of a prevention program for hamstring strain injury on optimum length of the knee flexors," in IOC World Conference on Prevention of Injury \& Illness in Sport (Monaco: British Journal of Sports Medicine), 633.

Mjolsnes, R., Arnason, A., Osthagen, T., Raastad, T., and Bahr, R. (2004). A 10 week randomized trial comparing eccentric vs. concentric hamstring strength training in well-trained soccer players. Scand. J. Med. Sci. Sports 14, 311-317. doi: 10.1046/j.1600-0838.2003.367.x

Moritani, T., and deVries, H. A. (1979). Neural factors versus hypertrophy in the time course of muscle strength gain. Am. J. Phys. Med. 58, 115-130.

Murphy, J. C., O’Malley, E., Gissane, C., and Blake, C. (2012). Incidence of injury in Gaelic football: a 4-year prospective study. Am. J. Sports Med. 40, 2113-2120. doi: $10.1177 / 0363546512455315$

Nichols, A. W. (2013). Does eccentric training of hamstring muscles reduce acute injuries in soccer? Clin. J. Sport Med. 23, 85-86. doi: 10.1097/JSM.0b013e318 $27 \mathrm{e} 9 \mathrm{f} 40$

Noorkoiv, M., Nosaka, K., and Blazevich, A. J. (2014). Neuromuscular adaptations associated with knee joint angle-specific force change. Med. Sci. Sports Exerc. 46, 1525-1537. doi: 10.1249/MSS.0000000000000269

Novacheck, T. F. (1998). The biomechanics of running. Gait Posture 7, 77-95. doi: 10.1016/S0966-6362(97)00038-6

Opar, D. A., Williams, M. D., and Shield, A. J. (2012). Hamstring strain injuries: factors that lead to injury and re-injury. Sports Med. 42, 209-226. doi: 10.2165/11594800-000000000-00000

Orchard, J. W., Seward, H., and Orchard, J. J. (2013). Results of 2 decades of injury surveillance and public release of data in the Australian football league. Am. J. Sports Med. 41, 734-741. doi: 10.1177/0363546513476270

Petersen, J., Thorborg, K., Nielsen, M. B., Budtz-Jorgensen, E., and Holmich, P. (2011). Preventive effect of eccentric training on acute hamstring injuries in men's soccer: a cluster-randomized controlled trial. Am. J. Sports Med. 39, 2296-2303. doi: 10.1177/0363546511419277

Potier, T. G., Alexander, C. M., and Seynnes, O. R. (2009). Effects of eccentric strength training on biceps femoris muscle architecture and knee joint range of movement. Eur. J. Appl. Physiol. 105, 939-944. doi: 10.1007/s00421-008-0980-7

Reeves, N. D., Maganaris, C. N., Longo, S., and Narici, M. V. (2009). Differential adaptations to eccentric versus conventional resistance training in older humans. Exp. Physiol. 94, 825-833. doi: 10.1113/expphysiol.2009. 046599

Reeves, N. D., Narici, M. V., and Maganaris, C. N. (2004). In vivo human muscle structure and function: adaptations to resistance training in old age. Exp. Physiol. 89, 675-689. doi: 10.1113/expphysiol.2004.027797

Schache, A. G., Dorn, T. W., Blanch, P. D., Brown, N. A., and Pandy, M. G. (2012). Mechanics of the human hamstring muscles during sprinting. Med. Sci. Sports Exerc. 44, 647-658. doi: 10.1249/MSS.0b013e318236a3d2

Seger, J. Y., Arvidsson, B., and Thorstensson, A. (1998). Specific effects of eccentric and concentric training on muscle strength and morphology in humans. Eur. J. Appl. Physiol. Occup. Physiol. 79, 49-57. doi: 10.1007/s004210 050472

Seynnes, O. R., de Boer, M., and Narici, M. V. (2007). Early skeletal muscle hypertrophy and architectural changes in response to high-intensity resistance training. J Appl Physiol 102, 368-373. doi: 10.1152/japplphysiol.0078 9.2006

Thelen, D. G., Chumanov, E. S., Hoerth, D. M., Best, T. M., Swanson, S. C., Li, L., et al. (2005). Hamstring muscle kinematics during treadmill sprinting. Med. Sci. Sports Exerc. 37, 108-114. doi: 10.1249/01.MSS.0000150078.79120.C8

Timmins, R. G., Ruddy, J. D., Presland, J., Maniar, N., Shield, A. J., Williams, M. D., et al. (2016a). Architectural changes of the biceps femoris long head after concentric or eccentric training. Med. Sci. Sports Exerc. 48, 499-508. doi: 10.1249/MSS.0000000000000795 
Timmins, R. G., Shield, A. J., Williams, M. D., Lorenzen, C., and Opar, D. A. (2016b). Architectural adaptations of muscle to training and injury: a narrative review outlining the contributions by fascicle length, pennation angle and muscle thickness. Br. J. Sports Med. doi: 10.1136/bjsports-2015-094881

Tomberlin, J. P., Basford, J. R., Schwen, E. E., Orte, P. A., Scott, S. C., Laughman, R. K., et al. (1991). Comparative study of isokinetic eccentric and concentric quadriceps training. J. Orthop. Sports Phys. Ther. 14, 31-36. doi: 10.2519/jospt.1991.14.1.31

Warren, G. L., Ingalls, C. P., Lowe, D. A., and Armstrong, R. B. (2001). Excitationcontraction uncoupling: major role in contraction-induced muscle injury. Exerc. Sport Sci. Rev. 29, 82-87. doi: 10.1097/00003677-200104000-00008

Woodley, S. J., and Mercer, S. R. (2005). Hamstring muscles: architecture and innervation. Cells Tissues Organs 179, 125-141. doi: 10.1159/0000 85004
Conflict of Interest Statement: The authors declare that the research was conducted in the absence of any commercial or financial relationships that could be construed as a potential conflict of interest.

The reviewer AN and handling Editor declared their shared affiliation, and the handling Editor states that the process nevertheless met the standards of a fair and objective review.

Copyright (C) 2016 Guex, Degache, Morisod, Sailly and Millet. This is an open-access article distributed under the terms of the Creative Commons Attribution License (CC $B Y)$. The use, distribution or reproduction in other forums is permitted, provided the original author(s) or licensor are credited and that the original publication in this journal is cited, in accordance with accepted academic practice. No use, distribution or reproduction is permitted which does not comply with these terms. 